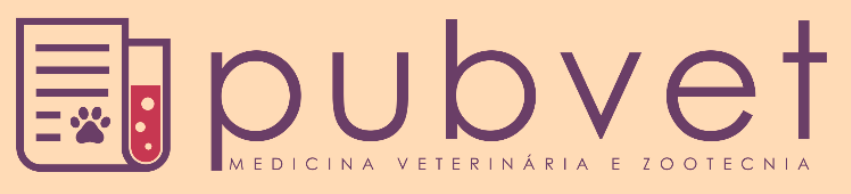

https://doi.org/10.31533/pubvet.v15n2a751.1-1 1

\title{
O uso de óleos essenciais no tratamento da malassezíase de cães e gatos: Revisão
}

\author{
Cecilia Andrade Sousa ${ }^{1} \bullet$ (D), Sávio Matheus Reis de Carvalho ${ }^{\bullet}$, Jean Rodrigues Carvalhoº \\ Silvana Maria Medeiros de Sousa Silva ${ }^{3} \bullet$ (D), Napoleão Martins Argôlo Neto ${ }^{3} \bullet$ (D)*
}

${ }^{I}$ Discente da Universidade Federal do Piauí, Programa de Pós-Graduação em Tecnologia Aplicadas a Animais de Interesse Regional. Teresina-PI Brasil. ${ }^{2}$ Médico Veterinário graduado pela Universidade Federal do Piauí. Bom Jesus-PI, Brasil.

${ }^{3}$ Professor adjunto da Universidade Federal do Piauí, Programa de Pós-Graduação em Tecnologia Aplicadas a Animais de Interesse Regional. Teresina-PI, Brasil. *Autor para correspondência, E-mail: argolo_napoleao@ufpi.edu.br

Resumo. A malassezíase é uma dermatopatia fúngica comum de humanos e animais, causada pela levedura lipofílica Malassezia pachydermatis. $\mathrm{O}$ tratamento baseia-se na associação entre antifúngico sistêmico e tópico, contudo, recidivas são frequentes e complicadas por dermatoses intercorrentes que complicam o caso clínico. Essa recorrência comumente deve-se a erros de posologia dos fármacos, de administração dos mesmos por proprietários e por resistência da levedura. Os mecanismos de resistência antifúngica estão associados às características específicas ou adquiridas do patógeno aos fármacos. Como alternativa aos tratamentos tradicionais, o uso de produtos fitoterápicos, como óleos essenciais, na clínica veterinária vem se popularizando. Embora a possível ação dos óleos essenciais seja referida em medicina humana há cerca de 17 anos, a literatura especializada sobre seu uso na malassezíase canina e felina ainda é escassa e carecem estudos que investiguem os mecanismos de ação de óleos essenciais no metabolismo da levedura Malassezia pachydermatis. Desta forma, o objetivo deste trabalho é apresentar uma revisão bibliográfica sobre o uso de quatro óleos essenciais para o tratamento da malassezíase primária ou secundária em cães e gatos.

Palavras chaves: Fitoterapia animal, malasseziose, óleos essenciais, otite externa

\section{The use of essential oils in the treatment of malasseziasis in dogs and cats: Review}

\begin{abstract}
Malasseziasis is a common fungal skin disease of humans and animals, caused by the lipophilic yeast Malassezia pachydermatis. Treatment is based on the association between systemic and topical antifungal; however, recurrences are frequent and complicated by intercurrent dermatoses that complicate the clinical case. This recurrence is usually due to errors in dosage of the drugs, administration of the same by owners and resistance of the yeast. The mechanisms of antifungal resistance are associated with the specific or acquired characteristics of the pathogen to the drugs. As an alternative to traditional treatments, the use of herbal products, such as essential oils, in the veterinary clinic has become popular. Although the possible action of essential oils has been reported in human medicine for about 17 years, the specialized literature on its use in canine and feline malasseziasis is still scarce and studies are needed to investigate the mechanisms of action of essential oils in the metabolism of the yeast Malassezia pachydermatis. Thus, the objective of this work is to present a bibliographic review on the use of four essential oils for the treatment of primary or secondary malasseziasis in dogs and cats.
\end{abstract}

Keywords: Animal phytotherapy, essential oils, external otitis, malasseziosis 


\section{El uso de aceites esenciales en el tratamiento de malasseziasis en perros y gatos: Revisión}

Resumen. La malasseziasis es una enfermedad cutánea fúngica común en humanos y animales, causada por la levadura lipofílica Malassezia pachydermatis. El tratamiento se basa en la asociación entre antifúngico sistémico y tópico, sin embargo, las recidivas son frecuentes y complicadas por dermatosis intercurrentes que complican el caso clínico. Esta recurrencia se debe comúnmente a errores en la dosificación de los medicamentos, administración de estos por parte de los propietarios y resistencia de la levadura. Los mecanismos de resistencia a los antifúngicos están asociados con las características específicas o adquiridas del patógeno a los fármacos. Como alternativa a los tratamientos tradicionales, se ha popularizado en la clínica veterinaria el uso de productos a base de hierbas, como los aceites esenciales. Aunque la posible acción de los aceites esenciales se ha mencionado en la medicina humana durante unos 17 años, la literatura especializada sobre su uso en malasseziasis canina y felina es aún escasa y se necesitan estudios para investigar los mecanismos de acción de los aceites esenciales en el metabolismo de la levadura Malassezia pachydermatis. Así, el objetivo de este trabajo es presentar una revisión bibliográfica sobre el uso de cuatro aceites esenciales para el tratamiento de malasseziasis primaria o secundaria en perros y gatos.

Palabras clave: Aceites esenciales, fitoterapia animal, malasseziosis, otitis externa

\section{Introdução}

A aromaterapia foi instituída por René-Maurice Gatefossé na década de 1920 para aplicações em tratamento de várias doenças na medicina (Sharifi-Rad et al., 2017). Considera-se que os óleos essenciais (OEs) são substâncias complexas, voláteis e de fragrância variável, provenientes de qualquer parte da planta, e resultantes do metabolismo secundário das plantas aromáticas (Brito et al., 2013). Além disso, podem apresentar diversas propriedades como antimicrobianas e antifúngicas. Essas funções foram avaliadas por meio de um ensaio contra cepas bacterianas e fúngicas de diversos gêneros, incluindo Malassezia (Artini et al., 2018).

A Malassezia pachydermatis é uma levedura lipofílica, comensal da pele e de mucosas de cães. Como patógeno secundário oportunista é responsável por otites nesses animais (Bond et al., 2020; Guillot \& Bond, 2020). O tratamento específico é baseado em aplicações de medicamento antifúngico, por via sistêmica e tópica, combinado com tratamento adjuvante com antibióticos para controlar infecção bacteriana secundária (Bismarck et al., 2020). Essa doença é caracterizada por recidivas, e um estudo recente in vitro descreveu que leveduras cultivadas a partir de lesões clínicas podem ser mais resistentes a drogas antifúngicas, quando conferidas a organismos procedentes de indivíduos assintomáticos (Ebani et al., 2020). Apesar de escassos, estes estudos recentes sugerem possível atividade antimicrobiana contra a Malassezia pachydermatis.

Assim, objetivou-se nesta revisão apresentar e discutir quatro fitoterápicos referidos como potencialmente efetivos para o tratamento da malassezíase na clínica de pequenos animais. Os OEs apresentados foram escolhidos baseado na frequência que foram citados em artigos científicos e pelo registro das plantas de obtenção na farmacopeia brasileira. Os dados foram obtidos nas bases PubMed, SciELO, ScinceDirect, Sage journals, Europe PMC, Google Scholar, Ovid, Scopus e ABFIT. As palavras-chave utilizadas foram "Malassezia", "otites", "antifúngicos", "óleos essenciais" combinadas às palavras "cães", "gatos", "pequenos animais" e "animais de companhia", em português e inglês, nos últimos 20 anos.

\section{Óleos essenciais e a legislação brasileira}

Pela legislação brasileira, OEs são produtos voláteis de origem vegetal adquirido por meio de método físico, como destilação por arraste com vapor de água, destilação a pressão reduzida ou outro método adequado. Ademais, os OEs podem ser isolados ou combinados entre si, retificados, desterpenados ou concentrados. Compreende-se por retificados, os produtos que são submetidos a um processo de 
destilação fracionada para concentrar determinados componentes; por concentrados, os que foram parcialmente desterpenados; por desterpenados, aqueles foram removidos quase a totalidade dos terpenos (ANVISA, 2010). Os OEs são considerados como produtos fitoterápicos, por condizer na descrição de produto adquirido de planta medicinal e seus derivados, exceto substâncias isoladas, com o objetivo profilático, paliativo ou curativo. Como derivado vegetal é entendido como o produto da extração da planta medicinal in natura, podendo ocorrer na forma de extrato, tintura, óleo fixo e volátil, cera, exsudado e outros (Brasil, 2011).

No Brasil, as atividades ligadas a fitoterapia estão associadas às culturas ancestrais e a medicina popular por todas as regiões brasileiras (Ribeiro, 2019). A Organização Mundial da Saúde reconheceu oficialmente, em 1978, a utilização de fitoterápicos. A política de plantas medicinais e fitoterápicos em território nacional está presente desde 1981 regulamentada pela Portaria $\mathrm{n}^{\circ} 212 \mathrm{de} 11$ de setembro do Ministério da Saúde, que delibera sobre a pesquisa utilizando plantas medicinais na rotina clínica. Assim, em 1982, o Ministério da Saúde lançou o Programa de Pesquisa de Plantas Medicinais da Central de Medicamentos no intuito de promover o desenvolvimento de terapêuticas alternativas pautadas no valor farmacológico de preparações de uso popular, à base de plantas medicinais (Brasil, 2011).

A Farmacopeia Brasileira é considerada o Código Oficial Farmacêutico do país, onde estão instituídos os critérios de qualidade dos medicamentos em uso, sejam manipulados ou industrializados, compondo o conjunto de normas e monografias de farmacoquímicos. Como integrante da Comissão da Farmacopeia Brasileira, o Comitê Técnico Temático de Apoio a Políticas de Plantas Medicinais e Fitoterápicos foi instituído para apoiar a implantação e implementação da Política Nacional de Plantas Medicinais e Fitoterápicos. Essa Política, aprovada através do Decreto $\mathrm{N}^{\circ} 5.813$, de 22 de junho de 2006, constitui diretrizes e linhas que priorizam o desenvolvimento de ações pelos inúmeros parceiros em torno de objetivos comuns voltados à garantia do acesso seguro e uso racional de plantas medicinais e fitoterápicos em nosso país, conforme a legislação vigente (ㅁasil, 2006).

$\mathrm{Na}$ farmacopeia brasileira podem ser encontradas diversas espécies vegetais importantes com embasamento científico para potencial uso terapêutico, as quais são apresentadas também para serem usadas no Sistema Único de Saúde (SUS). Observando isso, podem ser aproveitados esses fitoterápicos usados da medicina humana para aplicação na medicina veterinária.

\section{Resistência fúngica e efeito antifúngico de óleos essenciais}

Os OEs são metabólitos secundários de plantas que tem a função de proteção antimicrobiana e antiparasitária contra patógenos e insetos ambientais. Nas plantas, estes compostos podem ser secretados e armazenados em tricomas glandulares, pelos epidérmicos modificados, bolsas, reservatórios e espaços intercelulares. Além dos efeitos antimicrobianos, os OEs também podem apresentar benefícios para o vegetal quando atraem insetos importantes na dispersão de sementes e pólen (Ali et al., 2015; $\underline{\text { Sharifi- }}$ Rad et al., 2017).

Industrialmente, os OEs podem ser obtidos por processos de destilação de água ou vapor, destilação a seco do próprio material vegetal ou por processamento mecânico (Patra \& Saxena, 2010; Raeissi \& Peters, 2005). Caracterizados ainda, como compostos naturais complexos, lipofílicos e voláteis representados por constituintes químicos pertencentes a várias classes de compostos. Contudo, as classes mais encontradas são os terpenos e fenilpropanoides (Veloso et al., 2014). Os terpenos são quimicamente considerados como "alcenos naturais", isto significa que possuem uma dupla ligação carbono-carbono sendo caracterizado como um hidrocarboneto insaturado (Felipe \& Bicas, 2017). Além disso, os terpenos são reconhecidos por apresentarem atividade antimicrobiana (Lufti \& Roque, 2014). Já a segunda classe citada é derivada do ácido transcinâmico formado da fenilalanina em uma reação catalisada pela fenilalanina amônio liase (PAL). A atividade da PAL em plantas se ela por ocasião de estresses bióticos e abióticos e muitos fenilpropanoides são produzidos (Dixon \& Paiva, 1995).

As infecções ocasionadas por Malassezia spp na clínica veterinária são tratadas de forma frequente com o uso de derivados tópicos do azol (Deegan et al., 2019). No entanto, observou-se que essas recidivas são recorrentes (Oliveira et al., 2008). Essa recorrência está relacionada à prováveis erros de prescrição, administração de fármacos e resistência da levedura contra os medicamentos. Os mecanismos de resistência antifúngica estão associados às características específicas ou adquiridas do 
patógeno fúngico, que intervêm no mecanismo antifúngico da referida droga ou que diminuem os níveis de droga alvo (Peano et al., 2020).

Ademais, não está estabelecido um teste padrão de suscetibilidade antifúngica para $M$. pachydermatis; porém, estudos recentes revelaram que essa levedura, isolada de animais apresentando lesões de dermatite, demonstrou menor suscetibilidade a alguns agentes fúngicos em comparação com isolados de animais saudáveis (Bismarck et al., 2020; Jesus et al., 2011; Nijima et al., 2011). A demonstração de isolados de $M$. pachydermatis que respondem fracamente aos azóis justificam a importância de procurar tratamentos alternativos para o tratamento da malassezíase (Deegan et al., 2019). Contudo, a relação entre atividade dos OEs contra $M$. pachydermatis isolada de cães e gatos é escassa. Identificou-se uma pesquisa contemporânea in vitro investigando a atividade de doze OEs contra cepa de $M$. pachydermatis e de outros patógenos (Rusenova \& Parvanov, 2009). Outra pesquisa avaliou dezoito isolados de $M$. pachydermatis de orelhas de cães saudáveis e uma cepa de referência, tratados com 14 tipos de OEs (Váczi et al., 2018). Vinte e dois OEs foram testados quanto à sua atividade in vitro contra quinze isolados de $M$. pachydermatis de orelhas caninas. Os OEs foram testados e a atividade antifúngica volátil foi analisada pelo ensaio de vapor (Bismarck et al., 2020). Já em pesquisas in vivo, um estudo avaliou a efetividade de uma única mistura de $\mathrm{OE}$ (Kim et al., 2009) e outro, o efeito clínico de alguns OEs para tratamento da otite externa (Neves et al., 2018).

\section{Óleos essenciais referidos como potencialmente aplicáveis à medicina veterinária}

\section{- Óleo de Canela (Cinnamomum verum J. Presl)}

O óleo de Cinnamomum verum é conhecida popularmente como canela, usada frequentemente na elaboração de vários medicamentos na ciência farmacêutica (Koketsu et al., 1997). Os principais elementos que fazem parte das propriedades da planta são a emenagoga, estomáquica e carminativa (Cheng et al., 2009). O óleo é rico em cinamaldeído, o qual possui em sua composição fenólicos hidrofóbicos e terpenóides que desencadeiam uma importante atividade antimicrobiana in vitro, como bactérias Gram-positivas, Gram-negativas e fungos (Figueiredo et al., 2018). O cinamaldeído atua desorganizando a permeabilidade da membrana citoplasmática da célula e por consequência, inibe o crescimento do microoganismo (Taguchi et al., 2012).

Outros componentes antimicrobianos referidos são o eugenol (Devi et al., 2010) e linalol (Lorenzi et al., 2002). Sobre o eugenol, sabe-se que é um composto fenólico, constituinte de óleos essenciais considerado como agente fungicida (Hemaiswarya \& Doble, 2009). Essa ação de inibição microbiana do eugenol pode estar relacionada com a ruptura da membrana ou por inativação de enzimas e materiais genéticos (Oliveira \& Abreu Filho, 2012). O Linalol um elemento que demonstra efeitos antimicótico in vitro, devido ao mecanismo de desnaturação das proteínas ou desidratação sobre as células vegetativas (Cristina Figueiredo et al., 2001; Mundina et al., 1998).

Demonstrou-se em um estudo recente que o óleo essencial de canela apresentou atividade antifúngica mostrando graus de eficácia contra isolados de $M$. pachydermatis e revelando-se ativo com Concentração Inibitória Mínima (CIM) com 3,06 e 4,08 mg/mL (Ebani et al., 2020). Uma CIM corresponde à última diluição dos óleos essenciais, significando que não foi observada a presença de precipitado microbiano após o período de incubação, sendo, portanto a concentração em $\mathrm{mg} \cdot \mathrm{mL}^{-1}$ capaz de impedir o crescimento microbiano (Freire et al., 2014).

Analogamente, demonstrou-se sensibilidade da Malassezia spp a C. verum isolados do pavilhão auricular canino (Bismarck et al., 2020). Além disso, referiu-se atividade antifúngica do óleo essencial de C. verum (Chinh et al., 2017; Nagy et al., 2014). Outros autores referiram à ação antimicótica do óleo essencial de Cinnamomum verum em outras cepas, como de Fusarium responsáveis por ceratite em humanos e mostrou eficácia em uma concentração entre 31,25 e $500 \mu \mathrm{g} / \mathrm{mL}$, dependendo da cepa estudada (Chinh et al., 2017). Outra pesquisa ainda, concluiu que o óleo essencial de canela inibe o crescimento de Aspergillus flavus com uma CIM de 100ppm e tem uma ação antimicotoxigênica (Manso et al., 2011). 


\section{- Óleo de aroeira (Schinus terebinthifolius Raddi)}

$\mathrm{O}$ óleo essencial de aroeira é rico em monoterpenos, germacreno $\mathrm{D}$ e $(E)$ - $\beta$-cariofileno, os quais são responsáveis por várias atividades desta planta, sobretudo como agente antimicrobiano, atuando potencialmente, contra vários tipos de fungos (Santos et al., 2013; Silva et al., 2011; Znini et al., 2011).

Essa planta possui substâncias com referidas ação inseticida (Santos et al., 2013), anti-inflamatória (Medeiros et al., 2007), antimicrobiana (Gomes et al., 2013; Machado et al., 2012), antineoplásico (Queires et al., 2013), antifúngica (Alves et al., 2009; Azevedo et al., 2015; Freires et al., 2011; Nerio et al., 2010) e cicatrizantes (Azevedo et al., 2015). Identificou-se também relatos de tratamentos com animais utilizando óleo de aroeira a diminuição na sintomatologia clínica, manifestada inicialmente pelos animais, como prurido, dor e secreção (Nascente et al., 2004; Prashar et al., 2003).

$\mathrm{O}$ efeito antimicótico do óleo essencial de aroeira foi referido diante de alguns agentes etiológicos prevalentes na infecção otológica em cães (Nascente et al., 2004), Candida albicans, Aspergillus niger e Aspergillus flavus (Freires et al., 2011; Gundidza et al., 2009).

\section{- Alecrim (Rosmarinus officinalis)}

$\mathrm{O}$ alecrim pertencente à família Lamiaceae, de origem Mediterrânea e é referido como agente terapêutico potencial contra vários patógenos (Celiktas et al., 2007; Kabouche et al., 2005). Além disso, demonstra potencial in vitro de uso como antimicrobiano natural (Reis et al., 2020). Para a obtenção do óleo essencial do alecrim é usado às folhas e as sumidades floridas da planta (Carvalho \& Almança, 2003). No entanto, o Rosmarinus officinalis também pode ser usado in natura, na aromatização de formulações para o uso oral ou no preparo de infusões (Hentz \& Santin, 2007).

De modo geral, o óleo de alecrim é composto de várias substâncias como hidrocarbonetos monoterpênicos, ésteres terpênicos, linalol, verbinol, terpineol, 3-octanona e acetato de isobornila e por terpenoides, entre outros (Almela et al., 2006; Erkan et al., 2008; Sacchetti et al., 2005; Yosr et al., 2013).

Sobre a ação fungicida, verificou-se valores de Concentração Inibitória Mínima (CIM) e Concentração Fungicida Mínima (CFM) que variaram de $\leq 3,52$ a 112,8 mg para o óleo de alecrim frente a isolados de Malassezia pachydermatis. Verificou-se também que in vitro foi possível avaliar atividade antifúngica frente às leveduras isoladas de animais (Machado et al., 2013; Sousa et al., 2013).

Este óleo essencial também mostra atividade antimicrobiana contra microrganismos diversos. Um estudo descreveu ação efetiva contra Botrytis cinérea e contra Fusarium sp, quando o ED50 (dose de fungicida suficiente para inibir 50\% do crescimento micelial) determinado para este óleo essencial foi de aproximadamente $600 \mu \mathrm{g} / \mathrm{mL}$ e $660 \mu \mathrm{g} / \mathrm{mL}$, respectivamente. Além disso, 24 a $31 \mu \mathrm{g} / \mathrm{mL}$ foram suficientes para inibir 80\% do crescimento de Candida albicans (D'agostino et al., 2019). Outros estudos demonstraram uma CIM elevada contra Candida albicans (Almela et al., 2006; Erkan et al., 2008; Sacchetti et al., 2005; Yosr et al., 2013).

\section{- Capim limão (Cympopogon citratus)}

A espécie Cymbopogon citratus pertence à família Gramineae. Este vegetal é uma erva perene, com folhas estreitas e de alto valor comercial. Tem sido amplamente estudada, porque apresenta atividade antifúngica, antibacteriana, anti-helmíntica, inseticida, entre outros sendo estas propriedades atribuídas aos óleos voláteis a-citral, b-citral e mirceno (Almeida et al., 2013; Guimarães et al., 2011; Mohamed Hanaa et al., 2012; Regnier \& Combrinck, 2010).

Sobre a composição do $\mathrm{OE}$ de $C$. citratus, destacam-se os monoterpenos oxigenados $88,2 \%$ (Ebani et al., 2020). Os monoterpenos pertencem à classe dos terpenóides dos produtos naturais e são biosintetizados pela via do ácido mevalônico. Seu pequeno peso molecular, juntamente com alta natureza apolar, os torna os componentes mais abundantes dos óleos essenciais, que costumam ser considerados como tendo alguns efeitos antioxidantes e antimicrobianos gerais em concentrações razoavelmente altas (Habtemariam, 2018; Thoppil \& Bishayee, 2011). 
De modo geral, o óleo essencial de Cymbopogon citratus tem sido alvo de estudos em função do potencial antimicrobiano. A eficácia in vitro de OE contra o crescimento de M. pachydermatis mostrou diferentes graus e pode ser arranjada pela diminuição da efetividade. O capim-limão também inibiu o crescimento total de $M$. pachydermatis na placa de ágar em 14 de 15 casos. Verificou-se ainda, que em uma solução de $20 \%$ o OE de capim-limão apresentou um efeito inibidor do crescimento em $M$. pachydermatis (Bismarck et al., 2020; Nóbrega, 2019). Um estudo contemporâneo indicou que o potencial efeito antifúngico avaliado do óleo essencial de $C$. citratus pode estar relacionado à presença do terpeno citral, uma vez que, o composto mirceno só potencializou seus efeitos antimicrobianos quando combinados (Silva et al., 2009). A atividade desse componente foi investigada também em cepas do gênero Candida isoladas de infecções hospitalares. O óleo essencial de $C$. citratus apresentou atividade antifúngica em $100 \%$ dos isolados a partir da concentração de $25 \%$ (v/v), o que revela sua ação positiva sobre as cepas hospitalares (Almeida et al., 2013; Silva et al., 2009).

O óleo essencial de capim-limão foi avaliado também contra os fungos patogênicos Colletotrichum coccodes, Botrytis cinerea, Cladosporium herbarum, Rhizopus stolonifer e Aspergillus niger, in vitro, em que se verificou uma diminuição significativa do crescimento de todos os fungos (Tzortzakis \& Economakis, 2007).

\section{Considerações finais}

A literatura médica veterinária sobre a utilização e eficácia dos óleos essenciais para tratamento da malassezíase em cães e gatos ainda é escassa, predominando estudo in vitro ou estudos clínicos direcionados. Não se identificou nenhum estudo randomizado, duplo-cego abordando aspectos celulares, moleculares e clínicos. Contudo, a literatura disponível indica potencial efeito terapêutico antimicrobiano e ausência de efeitos colaterais.

Os óleos essenciais podem ser potenciais agentes terapêuticos, promissores para o tratamento de otite externa associada a $M$. pachydermatis. Contudo, dada diversidade e complexidade de composição dos vários óleos essenciais referidos com propriedade antifúngicos, faz-se mister ampliar as pesquisas ex vivo em animais, que elucidem o mecanismo de ação destas substâncias.

Considerando-se a referida resistência das leveduras pertencentes ao gênero Malassezia frente aos antifúngicos atualmente utilizados no Brasil, a identificação e estudo de novos agentes terapêuticos, como os óleos essenciais, assume importância salutar para a medicina veterinária.

\section{Referências}

Ali, B., Al-Wabel, N. A., Shams, S., Ahamad, A., Khan, S. A., \& Anwar, F. (2015). Essential oils used in aromatherapy: A systemic review. Asian Pacific Journal of Tropical Biomedicine, 5(8), 601-611. DOI: https://doi.org/10.1016/j.apjtb.2015.05.007

Almeida, R. B. A., Carretto, C. F. P., Santana, R. S., Furlan, M. R., Junqueira, J. C., \& Jorge, A. O. C. (2013). Atividade antimicrobiana de Cymbopogon citratus (DC.) stapf sobre Candida spp. Revista de Odontologia Da UNESP, 37(2), 147-153. https://doi.org/10.1590/S1516-05722013000400002.

Almela, L., Sánchez-Muñoz, B., Fernández-López, J. A., Roca, M. J., \& Rabe, V. (2006). Liquid chromatograpic-mass spectrometric analysis of phenolics and free radical scavenging activity of rosemary extract from different raw material. Journal of Chromatography A, 1120(1-2), 221-229. DOI: https://doi.org/10.1016/j.chroma.2006.02.056

Alves, P. M., Queiroz, L. M. G., Pereira, J. V., \& Pereira, M. S. V. (2009). Atividade antimicrobiana, antiaderente e antifúngica in vitro de plantas medicinais brasileiras sobre microrganismos do biofilme dental e cepas do gênero Candida. Revista Da Sociedade Brasileira de Medicina Tropical, 42(2), 222-224. DOI: https://doi.org/10.1590/s0037-86822009000200028

ANVISA. (2010). Farmacopeia Brasileira (5th ed., Vol. 1). Agência Nacional de Vigilância Sanitária e Fundação Oswaldo Cruz.

Artini, M., Patsilinakos, A., Papa, R., Božović, M., Sabatino, M., Garzoli, S., Vrenna, G., Tilotta, M., Pepi, F., \& Ragno, R. (2018). Antimicrobial and antibiofilm activity and machine learning classification analysis of essential oils from different mediterranean plants against Pseudomonas 
aeruginosa. Molecules, 23(2), 482. DOI: https://doi.org/10.3390/molecules23020482

Azevedo, C. F., Quirino, Z. G. M., \& Bruno, R. L. A. (2015). Estudo farmacobotânico de partes aéreas vegetativas de aroeira-vermelha (Schinus terebinthifolius Raddi, Anacardiaceae). Revista Brasileira de Plantas Medicinais, 17(1), 26-35. DOI: https://doi.org/10.1590/1983-084x/11 090

Bismarck, D., Dusold, A., Heusinger, A., \& Müller, E. (2020). Antifungal in vitro activity of essential oils against clinical isolates of Malassezia pachydermatis from canine ears: a report from a practice laboratory. Complementary Medicine Research, 27(3), 143-154. DOI: https://doi.org/10.1159/000504316

Bond, R., Morris, D. O., Guillot, J., Bensignor, E. J., Robson, D., Mason, K. V, Kano, R., \& Hill, P. B. (2020). Biology, diagnosis and treatment of Malassezia dermatitis in dogs and cats Clinical Consensus Guidelines of the World Association for Veterinary Dermatology. Veterinary Dermatology, 31(1), 27-e4. DOI: https://doi.org/10.1111/vde.12834

Brasil. Agência Nacional de Vigilância Sanitária. (2011). Formulário de Fitoterápicos da Farmacopéia Brasileira / Agência Nacional de Vigilância Sanitária. Brasília: Anvisa, 1, 126.

Brasil. Ministério da Saúde. Secretaria de Ciência, Tecnologia e Insumos Estratégicos. (2006). Departamento de Assistência Farmacêutica. Política nacional de plantas medicinais e fitoterápicos / Ministério da Saúde, Secretaria de Ciência, Tecnologia e Insumos Estratégicos, Departamento de Assistência Farmacêutica. - Brasília: Ministério da Saúde.

Brito, A. M. G., Rodrigues, S. A., Brito, R. G., \& Xavier-Filho, L. (2013). Aromaterapia: da gênese a atualidade. Revista Brasileira de Plantas Medicinais, 15(4), 789-793. DOI: https://doi.org/10.1590/s1516-05722013000500021

Carvalho, J. C. T., \& Almança, C. C. J. (2003). Formulário de prescrição fitoterápica. Ed. Atheneu.

Celiktas, O. Y., Kocabas, E. E. H., Bedir, E., Sukan, F. V., Ozek, T., \& Baser, K. H. C. (2007). Antimicrobial activities of methanol extracts and essential oils of Rosmarinus officinalis, depending on location and seasonal variations. Food Chemistry, 100(2), 553-559. DOI: https://doi.org/10.1016/j.foodchem.2005.10.011

Cheng, S.-S., Liu, J.-Y., Huang, C.-G., Hsui, Y.-R., Chen, W.-J., \& Chang, S.-T. (2009). Insecticidal activities of leaf essential oils from Cinnamomum osmophloeum against three mosquito species. Bioresource Technology, 100(1), 457-464. DOI: https://doi.org/10.1016/j.biortech.2008.02.030

Chinh, H. V, Luong, N. X., Thin, D. B., Dai, D. N., Hoi, T. M., \& Ogunwande, I. A. (2017). Essential oils leaf of Cinnamomum glaucescens and Cinnamomum verum from Vietnam. American Journal of Plant Sciences, 8(11), 2712. DOI: https://doi.org/10.4236/ajps.2017.811182

Cristina Figueiredo, A., Miguel, M. G., Duarte, A. M. F., Barroso, J. G., \& Pedro, L. G. (2001). Essential oil composition of Thymus lotocephallus G. López \& R. Morales, collected during flowering and vegetative phases. Flavour and Fragrance Journal, 16(6), 417-421. DOI: https://doi.org/10.1002/ffj.1028

D'agostino, M., Tesse, N., Frippiat, J. P., Machouart, M., \& Debourgogne, A. (2019). Essential oils and their natural active compounds presenting antifungal properties. Molecules, 24(20), 3713. DOI: https://doi.org/10.3390/molecules24203713

Deegan, K. R., Fonseca, M. S., Oliveira, D. C. P., Santos, L. M., Hanna, S. A., Machado, B. A. S., Umsza-Guez, M. A., Meyer, R., \& Portela, R. W. (2019). Susceptibility of Malassezia pachydermatis clinical isolates to allopathic antifungals and brazilian red, green, and brown propolis extracts. Frontiers in Veterinary Science, 6, 460. DOI: https://doi.org/10.3389/fvets.2019.00460

Devi, K. P., Nisha, S. A., Sakthivel, R., \& Pandian, S. K. (2010). Eugenol (an essential oil of clove) acts as an antibacterial agent against Salmonella typhi by disrupting the cellular membrane. Journal of Ethnopharmacology, 130(1), 107-115. DOI: https://doi.org/10.1016/j.jep.2010.04.025

Dixon, R. A., \& Paiva, N. L. (1995). Stress-induced phenylpropanoid metabolism. The Plant Cell, 7(7), 1085. DOI: https://doi.org/10.2307/3870059

Ebani, V. V., Bertelloni, F., Najar, B., Nardoni, S., Pistelli, L., \& Mancianti, F. (2020). Antimicrobial activity of essential oils against Staphylococcus and Malassezia strains isolated from canine dermatitis. Microorganisms, 8(2), 252. DOI: https://doi.org/10.3390/microorganisms8020252 
Erkan, N., Ayranci, G., \& Ayranci, E. (2008). Antioxidant activities of rosemary (Rosmarinus Officinalis L.) extract, blackseed (Nigella sativa L.) essential oil, carnosic acid, rosmarinic acid and sesamol. Food Chemistry, 110(1), 76-82. DOI: https://doi.org/10.1016/j.foodchem.2008.01.058

Felipe, L. O., \& Bicas, J. L. (2017). Terpenos, aromas e a química dos compostos naturais. Química Nova Na Escola, 39(2), 120-130. DOI: https://doi.org/10.21577/0104-8899.20160068

Figueiredo, C., Oliveira, P. V, Silva, S. W., Muniz, D. R., Silva, R. J., Maia, S. N., Nascimento, S. L., \& Grisotto, G. (2018). Óleo essencial da Canela (Cinamaldeído) e suas aplicações biológicas. Revista de Investigação Biomédica, 9(2), 192-197. DOI: https://doi.org/10.24863/rib.v9i2.143

Freire, I. C. M., Pérez, A., Cardoso, A. M. R., Mariz, B., Almeida, L. F. D., Cavalcanti, Y. W., \& Padilha, W. W. N. (2014). Atividade antibacteriana de Óleos Essenciais sobre Streptococcus mutans e Staphylococcus aureus. Revista Brasileira de Plantas Medicinais, 16(2), 372-377. DOI: https://doi.org/10.1590/1983-084x/12 053

Freires, I. A., Alves, L. A., Jovito, V. C., \& Castro, R. D. (2011). Atividade antifúngica de Schinus terebinthifolius (Aroeira) sobre cepas do gênero Candida. Revista Odontológica Do Brasil Central, 20(52).

Gomes, F. S., Procópio, T. F., Napoleão, T. H., Coelho, L., \& Paiva, P. M. G. (2013). Antimicrobial lectin from S chinus terebinthifolius leaf. Journal of Applied Microbiology, 114(3), 672-679. DOI: $\underline{10.1111 / \mathrm{jam} .12086}$

Guillot, J., \& Bond, R. (2020). Malassezia yeasts in veterinary dermatology: an updated overview. Frontiers in Cellular and Infection Microbiology, 10, 79. DOI: https://doi.org/10.3389/fcimb.2020.00079

Guimarães, L. G. L., Cardoso, M. G., Sousa, P. E., Andrade, J., \& Vieira, S. S. (2011). Atividades antioxidante e fungitóxica do óleo essencial de capim-limão e do citral. Revista Ciência Agronômica, 42(2), 464-472. DOI: https://doi.org/10.1590/s1806-66902011000200028

Gundidza, M., Gweru, N., Magwa, M. L., Mmbengwa, V., \& Samie, A. (2009). The chemical composition and biological activities of essential oil from the fresh leaves of Schinus terebinthifolius from Zimbabwe. African Journal of Biotechnology, 8(24), 7164-7169. DOI: 10.4314/ajb.v8i24.68817

Habtemariam, S. (2018). Antidiabetic potential of monoterpenes: A case of small molecules punching above their weight. International Journal of Molecular Sciences, 19(1), 4. https://doi.org/10.3390/ijms19010004.

Hemaiswarya, S., \& Doble, M. (2009). Synergistic interaction of eugenol with antibiotics against Gram negative bacteria. Phytomedicine, 16(11), 997-1005. DOI: https://doi.org/10.1016/j.phymed.2009.04.006

Hentz, S. M. \& Santin, N. C. (2007). Avaliação da atividade antimicrobiana do óleo essencial de alecrim (Rosmarinus officinalis 1.) contra Salmonella sp. Evidencia, 7, 93-100

Jesus, F. P. K., Lautert, C., Zanette, R. A., Mahl, D. L., Azevedo, M. I., Machado, M. L. S., Dutra, V., Botton, S. A., Alves, S. H., \& Santurio, J. M. (2011). In vitro susceptibility of fluconazole-susceptible and-resistant isolates of Malassezia pachydermatis against azoles. Veterinary Microbiology, 152(12), 161-164. DOI: https://doi.org/10.1016/j.vetmic.2011.04.027

Kabouche, Z., Boutaghane, N., Laggoune, S., Kabouche, A., Ait-Kaki, Z., \& Benlabed, K. (2005). Comparative antibacterial activity of five Lamiaceae essential oils from Algeria. International Journal of Aromatherapy, 15(3), 129-133. DOI: https://doi.org/10.1016/j.ijat.2005.03.006

Kim, S.-H., Kim, S., Jun, H.-K., \& Kim, D.-H. (2009). Efficacy of aromatherapy for the treatment of otitis externa in dogs. Korean Journal of Veterinary Research, 49(1), 85-89.

Koketsu, M., Gonçalves, S. L., Godoy, R. L. O., Lopes, D., \& Morbach, N. (1997). Óleos essenciais de cascas e folhas de canela (Cinnamomum verum Presl) cultivada no Paraná. Sociedade Brasileira de Ciência e Tecnologia de Alimentos, 17. DOI: https://doi.org/10.1590/s0101-20611997000300017

Lorenzi, H., Matos, F. J., \& Francisco, J. M. (2002). Plantas medicinais no Brasil: nativas e exóticas (Vol. 1). 
Lufti, M., \& Roque, N. F. (2014). Histórias de Eugênias. Química Nova Na Escola, 36(4), 252-260. DOI: https://doi.org/10.5935/0104-8899.20140030

Machado, D. G., Cunha, M. P., Neis, V. B., Balen, G. O., Colla, A., Bettio, L. E. B., Oliveira, Á., Pazini, F. L., Dalmarco, J. B., Simionatto, E. L., Pizzolatti, M. G., \& Rodrigues, A. L. S. (2013). Antidepressant-like effects of fractions, essential oil, carnosol and betulinic acid isolated from Rosmarinus officinalis L. Food Chemistry, 136(2), 999-1005. DOI: https://doi.org/10.1016/j.foodchem.2012.09.028

Machado, J. A., Rebelo, M. A., Favaro, L. I. L., Vila, M. M. D. C., \& Gerenutti, M. (2012). In vitro evaluation of the antimicrobial potential association of Schinus terebinthifolius Raddi and Syzygium aromaticum L. Journal of Pharmacy, 2(3), 438-443. DOI: https://doi.org/10.9790/3013-0230438443

Manso, S., Nerin, C., \& Gómez-Lus, R. (2011). Antifungal activity of the essential oil of cinnamon (cinnamomum zeylanicum), oregano (origanum vulgare) and lauramide argine ethyl ester (LAE) against the mold aspergillus flavus CECT 2949. Italian Journal of Food Science, 23, 151.

Medeiros, K. C. P., Monteiro, J. C., Diniz, M. F. F. M., Medeiros, I. A., Silva, B. A., \& Piuvezam, M. R. (2007). Effect of the activity of the Brazilian polyherbal formulation: Eucalyptus globulus Labill, Peltodon radicans Pohl and Schinus terebinthifolius Radd in inflammatory models. Revista Brasileira de Farmacognosia, 17(1), 23-28. DOI: https://doi.org/10.1590/s0102$\underline{695 \times 2007000100006}$

Mohamed Hanaa, A. R., Sallam, Y. I., El-Leithy, A. S., \& Aly, S. E. (2012). Lemongrass (Cymbopogon citratus) essential oil as affected by drying methods. Annals of Agricultural Sciences, 57(2), 113116. DOI: https://doi.org/10.1016/j.aoas.2012.08.004

Mundina, M., Vila, R., Tomi, F., Gupta, M. P., Adzet, T., Casanova, J., \& Cañigueral, S. (1998). Leaf essential oils of three panamanian Piper species. Phytochemistry, 47(7), 1277-1282. DOI: https://doi.org/10.1016/s0031-9422(97)00762-0

Nagy, G., Hochbaum, T., Sarosi, S., \& Ladanyi, M. (2014). In vitro and in planta activity of some essential oils against Venturia inaequalis (Cooke) G. Winter. Notulae Botanicae Horti Agrobotanici Cluj-Napoca, 42(1), 109-114. DOI: https://doi.org/10.15835/nbha4219503

Nascente, P. S., Nobre, M. O., Meinerz, A. R. M., Gomes, F. R., Souza, L. L., \& Meireles, M. C. A. (2004). Ocorrência de Malassezia pachydermatis em cães e gatos. Revista Brasileira de Medicina Veterinária, 26(2), 79-82.

Nerio, L. S., Olivero-Verbel, J., \& Stashenko, E. (2010). Repellent activity of essential oils: A review. Bioresource Technology, 101(1), 372-378. DOI: https://doi.org/10.1016/j.biortech.2009.07.048

Neves, R. C. S. M., Makino, H., Cruz, T. P. P. S., Silveira, M. M., Sousa, V. R. F., Dutra, V., Lima, M. E. K. M., \& Belli, C. B. (2018). In vitro and in vivo efficacy of tea tree essential oil for bacterial and yeast ear infections in dogs. Pesquisa Veterinária Brasileira, 38(8), 1597-1607. DOI: https://doi.org/10.1590/1678-5150-pvb-5055

Nijima, M., Kano, R., Nagata, M., Hasegawa, A., \& Kamata, H. (2011). An azole-resistant isolate of Malassezia pachydermatis. Veterinary Microbiology, 149(1-2), 288-290. DOI: https://doi.org/10.1016/j.vetmic.2010.10.010

Nóbrega, L. K. de S. (2019). Óleos essenciais com efeito sobre Malassezia spp.: uma revisão integrativa. Revista de Bioclimatologia e Ciência, 8, 43-55.

Oliveira, J. G., \& Abreu Filho, B. A. (2012). Propriedade antimicrobiana do eugenol frente às amostras de Alicyclobacillus spp. isoladas de suco de laranja. Revista Do Instituto Adolfo Lutz, 71(2), 410 414.

Oliveira, L. C., Carvalho, C., Leite, C. A. L., \& Brilhante, R. S. N. (2008). Comparative study of the microbial profile from bilateral canine otitis externa. Canadian Veterinary Journal, 49(8), 785-788.

Patra, A. K., \& Saxena, J. (2010). A new perspective on the use of plant secondary metabolites to inhibit methanogenesis in the rumen. Phytochemistry, 7l(11-12), 1198-1222. DOI: https://doi.org/10.1002/chin.201041275

Peano, A., Johnson, E., Chiavassa, E., Tizzani, P., Guillot, J., \& Pasquetti, M. (2020). Antifungal resistance regarding Malassezia pachydermatis: Where are we now? Journal of Fungi, 6(2), 93. DOI: 


\section{https://doi.org/10.3390/jof6020093}

Prashar, A., Hili, P., Veness, R. G., \& Evans, C. S. (2003). Antimicrobial action of palmarosa oil (Cymbopogon martinii) on Saccharomyces cerevisiae. Phytochemistry, 63(5), 569-575. DOI: https://doi.org/10.1016/s0031-9422(03)00226-7

Queires, L. C., Crépin, M., Vacherot, F., de la Taille, A., \& Rodrigues, L. E. (2013). In vitro effects of polyphenols extracted from the aroeira plant (Schinus terebinthifolius Raddi) on the growth of prostate cancer cells (Lncap, Pc-3 and Du145). Brazilian Journal of Medicine and Human Health, 1(1), 71-82. DOI: https://doi.org/10.17267/2317-3386bjmhh.v1i1.114

Raeissi, S., \& Peters, C. J. (2005). Liquid-vapor and liquid-liquid-vapor equilibria in the ternary system ethane. The Journal of Supercritical Fluids, 33(3), 201-208. https://doi.org/http://dx.doi.org/10.1016/j.supflu.2004.08.007

Regnier, T., \& Combrinck, S. (2010). In vitro and in vivo screening of essential oils for the control of wet bubble disease of Agaricus bisporus. South African Journal of Botany, 76(4), 681-685. DOI: https://doi.org/10.1016/j.sajb.2010.07.018

Reis, J. B., Figueiredo, L. A., Castorani, G. M., \& Veiga, S. M. O. M. (2020). Avaliação da atividade antimicrobiana dos óleos essenciais contra patógenos alimentares/Evaluation of antimicrobial activity of essential oils against food pathogens. Brazilian Journal of Health Review, 3(1), 342-363. DOI: https://doi.org/10.35587/brj.ed.0000222

Ribeiro, L. H. L. (2019). Análise dos programas de plantas medicinais e fitoterápicos no Sistema Único de Saúde (SUS) sob a perspectiva territorial. Ciência \& Saúde Coletiva, 24(5), 1733-1742. DOI: https://doi.org/10.1590/1413-81232018245.15842017

Rusenova, N., \& Parvanov, P. (2009). Antimicrobial activities of twelve essential oils against microorganisms of veterinary importance. Trakia Journal of Sciences, 7(1), 37-43.

Sacchetti, G., Maietti, S., Muzzoli, M., Scaglianti, M., Manfredini, S., Radice, M., \& Bruni, R. (2005). Comparative evaluation of 11 essential oils of different origin as functional antioxidants, antiradicals and antimicrobials in foods. Food Chemistry, 91(4), 621-632. DOI: https://doi.org/10.1016/j.foodchem.2004.06.031

Santos, M. R. A., Lima, R. A., Silva, A. G., Lima, D. K. S., Sallet, L. A. P., Teixeira, C. A. D., \& Facundo, V. A. (2013). Composição química e atividade inseticida do óleo essencial de Schinus terebinthifolius Raddi (Anacardiaceae) sobre a broca-do-café (Hypothenemus hampei) Ferrari. Revista Brasileira de Plantas Medicinais, 15(4), 757-762. DOI: https://doi.org/10.1590/s1516$\underline{05722013000500017}$

Sharifi-Rad, J., Sureda, A., Tenore, G. C., Daglia, M., Sharifi-Rad, M., Valussi, M., Tundis, R., SharifiRad, M., Loizzo, M. R., \& Ademiluyi, A. O. (2017). Biological activities of essential oils: From plant chemoecology to traditional healing systems. Molecules, 22(1), $70 . \quad$ DOI: https://doi.org/10.3390/molecules22010070

Silva, F. S., Ferreira, T. M., Teodoro, G. R., Costa, A. C. B. P., Maria, A., Beltrame Junior, M., Salvador, M. J., Paula, C. R., \& Khouri, S. (2009). Antifungal activity of Cymbopogon citratus essential oil on Candida albicans and Candida tropicalis strains isolated from nosocomial infections. Revista Do Instituto Adolfo Lutz(Impresso), 68(3), 434-441.

Silva, M. A., Sousa, B. M. P., Zanini, S. F., Colnago, G. L., Nunes, L. C., Rodrigues, M. R. A., \& Ferreira, L. (2011). Óleo essencial de aroeira-vermelha como aditivo na ração de frangos de corte. Ciência Rural, 41(4), 676-681. DOI: https://doi.org/10.1590/s0103-84782011005000034

Sousa, L. L., Andrade, S. C. A., Athayde, A. J. A. A., Oliveira, C. E. V., Sales, C. V., Madruga, M. S., \& Souza, E. L. (2013). Efficacy of Origanum vulgare L. and Rosmarinus officinalis L. essential oils in combination to control postharvest pathogenic Aspergilli and autochthonous mycoflora in Vitis labrusca L. (table grapes). International Journal of Food Microbiology, 165(3), 312-318. DOI: https://doi.org/10.1016/j.ijfoodmicro.2013.06.001

Taguchi, Y., Hasumi, Y., Hayama, K., Arai, R., Nishiyama, Y., \& Abe, S. (2012). Effect of cinnamaldehyde on hyphal growth of $\mathrm{C}$. albicans under various treatment conditions. Medical Mycology Journal, 53(3), 199-204. DOI: https://doi.org/10.3314/mmj.53.199 
Thoppil, R. J., \& Bishayee, A. (2011). Terpenoids as potential chemopreventive and therapeutic agents in liver cancer. World Journal of Hepatology, 3(9), 228. DOI: https://doi.org/10.4254/wjh.v3.i9.228

Tzortzakis, N. G., \& Economakis, C. D. (2007). Antifungal activity of lemongrass (Cympopogon citratus L.) essential oil against key postharvest pathogens. Innovative Food Science \& Emerging Technologies, 8(2), 253-258. DOI: https://doi.org/10.1016/j.ifset.2007.01.002

Váczi, P., Čonková, E., Marcinčáková, D., \& Sihelská, Z. (2018). Antifungal effect of selected essential oils on Malassezia pachydermatis growth. Folia Veterinaria, 62(2), 67-72. DOI: https://doi.org/10.2478/fv-2018-0018

Veloso, R. A., Castro, H. G., Barbosa, L. C. A., Cardoso, D. P., Chagas Júnior, A. F., \& Scheidt, G. N. (2014). Teor e composição do óleo essencial de quatro acessos e duas cultivares de manjericão (Ocimum basilicum L.). Revista Brasileira de Plantas Medicinais, 16(2), 364-371. DOI: https://doi.org/10.1590/1983-084x/12 180

Yosr, Z., Hnia, C., Rim, T., \& Mohamed, B. (2013). Changes in essential oil composition and phenolic fraction in Rosmarinus officinalis L. var. typicus Batt. organs during growth and incidence on the antioxidant activity. Industrial Crops and Products, 43(0), 412-419. DOI: https://doi.org/10.1016/j.indcrop.2012.07.044

Znini, M., Cristofari, G., Majidi, L., Mazouz, H., Tomi, P., Paolini, J., \& Costa, J. (2011). Antifungal activity of essential oil from Asteriscus graveolens against postharvest phytopathogenic fungi in apples. Natural Product Communications, 6(11), 1763-1768. DOI: https://doi.org/10.1177/1934578x1100601147.

Recebido: 15 de agosto, 2020.

Aprovado: 10 de outubro, 2020.

Disponível online: 22 de dezembro, 2020.
Licenciamento: Este artigo é publicado na modalidade Acesso Aberto sob a licença Creative Commons Atribuição 4.0 (CC-BY 4.0), a qual permite uso irrestrito, distribuição, reprodução em qualquer meio, desde que o autor e a fonte sejam devidamente creditados. 